\title{
Control Performance Assessment of Fractional-Order PID Controllers Applied to Tracking Trajectory Control of Robotic Systems
}

\author{
LUIS ANGEL \\ Department of Electronic Engineering \\ Universidad Pontificia Bolivariana \\ Via Piedecuesta km 7, Bucaramanga, COLOMBIA
}

\author{
JAIRO VIOLA \\ Department of Mechanical Engineering \\ University of California, Merced \\ 5200 Lake Rd, Merced, CA 95343, USA
}

\begin{abstract}
Performing tracking tasks in robotic manipulators presents many challenges for the controller design, especially in presence of external disturbances that affects the dynamical behavior of the robotic system. This paper presents the design of a Fractional-Order PID controller with the computed torque control strategy for the tracking control of a two degree of freedom robotic manipulator. The proposed technique is contrasted against the classical PID controller with the computed torque control strategy. To validate the proposed controllers, the robotic system is simulated using an MSC-ADAMS/MATLAB co-simulation model, which is employed for identification and control tasks. The proposed model is tested in presence of external disturbances in the applied torque, random noise in the feedback loop and payload variations. Obtained results show that the Fractional-Order PID controller with the computed torque control strategy has a better performance in presence of the analyzed external disturbances for tracking tasks.
\end{abstract}

Key-Words: - Fractional control, FOPID, IOPID, MSC-ADAMS/MATLAB cosimulation model.

Received: March 16, 2021. Revised: January 3, 2022. Accepted: January 22, 2022. Published: February 10, 2022.

\section{Introduccion}

Robotic manipulators perform position and tracking tasks, using the dynamic model of the robotic system for the design of the control strategy. If the dynamic model of the manipulator is well known, the tracking tasks control can be designed using the computed torque control strategy. This control strategy employs the feedback linearization to compensate the nonlinearities of the robotic manipulator model, allowing the application of classic control strategies as the PID controller. Although this control strategy is simple and effective, rarely the complete dynamic model of the robotic system is available due to the presence of external disturbances and parametric uncertainness biasing the controller tuning and affecting the stability and performance of the system.

Control strategies like QFT [6][7], H infinity [8][9], adaptive control [10], [11], or $\mu$ synthesis [10][12] are employed for the control of robotic systems with external disturbances and parametric uncertainness. However, the main limitation of these control strategies is the lack of knowledge of the robotic system dynamics.

On the other hand, Fractional-Order control strategies can help to reach a robust performance in the presence of external disturbances and parametric uncertainness even without a complete dynamic model of the system [13], [14]-[16].

This paper presents the design of a tracking control for a two degree of freedom robotic manipulator employing a Fractional-Order PID controller (FOPID) with the computed torque control strategy. Initially, the kinematic and dynamic model of the robotic system is calculated. Then, to MSCADAMS / MATLAB cosimulation model of the manipulator is built for the stages of identification, control, and validation. After that, the dynamic model of the robot is identified employing the recursive least squares algorithm. From the 
identified dynamic model, the FOPID controller with computer torque control is tuned.

An Integer-Order PID controller (IOPID) is designed to compare the performance of the FOPID controller. Then, a robustness analysis is performed for the IOPID and FOPID controllers considering the presence of external disturbances in the joints torque, random noise in the feedback loop, and payload variations. The results of the robustness analyzed are quantified using a novel Control Performance Assessment methodology, which proposes a set of performance metrics based on the angular position, velocity, and acceleration errors of the manipulator.

The main contribution of this paper is the implementation of the Computed Torque Control strategy with the Fractional Order PID controller for the tracking trajectory problem applied to robotic systems and the development of a novel control performance assessment methodology for fractionalorder controllers.

This paper is structured as follows. Section II shows the computed torque control strategy and the design methodology for the IOPID and FOPID controllers with the computed torque control strategy. Section III presents the case study, a two degree of freedom robotic manipulator, its kinematic and the dynamic models, and the MSC-ADAMS/MATLAB cosimulation model and its parametric identification. Section IV presents the IOPID and FOPID controllers with computed torque control design and its performance for different trajectories. Section V shows the robustness tests and Control Performance Assessment for the FOPID and IOPID controllers. Finally, conclusions and future works are presented.

\section{Feedback linearization via Computed Torque Control}

Computed torque control (CTC) employs the feedback linearization technique to obtain a linearized and decoupled model of the robotic system to control it with linear control strategies.
From [17], the Dynamic model of a robotic system is given by (1).

$\tau=M(q) \ddot{q}+C(q, \dot{q})+g(q)$

where $q, \dot{q}, \ddot{q}$ are the manipulator joints position, velocity and acceleration, $M(q)$ is the inertia matrix, $C(q, \dot{q})$ is the Coriolis matrix, and $g(q)$ is the gravity vector. Applying the linearization law (2) where $a$ is the new input of the system.

$\tau=M(q) a+C(q, \dot{q})+g(q)$.

Assuming an exact knowledge of the dynamic model, combining (1) and (2) results (3).

$M(q) a=M(q) \ddot{q}$

If $M(q)$ is invertible, (3) correspond to a linearized and decoupled double integrator system for each link of the robotic system as shown in (4).

$\ddot{q}=a$

For the design of the IOPID controller, the control law (4) is proposed, where $\tilde{q}$ is the joint position error, $\tilde{\dot{q}}$ is the joint velocity error, and $\ddot{q_{d}}$ is the desired joint acceleration.

$a=\ddot{q}_{d}-k_{p} \tilde{q}-k_{i} \int \tilde{q} d t-k_{d} \tilde{\dot{q}}$.

Replacing (5) in (4), the control law in closed loop for the robotic system is given by (6).

$$
\tilde{\ddot{q}}+k_{p} \tilde{q}+k_{i} \int \tilde{q} d t+k_{d} \tilde{\dot{q}}=0
$$

Passing (6) to the Laplace Domain, the characteristic polynomial of the system is obtained.

$s^{2}+k_{p}+\frac{k_{i}}{s}+k_{d} s=0$

In order to find the IOPID controller terms, the desired polynomial is given by (8) where $\alpha$ is a nondominant pole of the closed system, $\zeta$ is the 
damping ratio, and $w_{n}$ is the natural frequency of the system.

$$
(s+\alpha)\left(s^{2}+2 \zeta w_{n} s+w_{n}^{2}\right)=0
$$

From (7) and (8), the resulting expression (9) allows to calculate the terms of the IOPID controller for each joint.

$k_{p}=w_{n}^{2}+2 \alpha \zeta w_{n}$

$k_{i}=\alpha w_{n}^{2}$

$k_{d}=2 \zeta w_{n}+\alpha$

According to [18]-[20], FOPID controller is defined by the following integral-differential equation (10).

$$
u(t)=k_{p} e(t)+k_{i} D^{-\lambda} e(t)+k_{d} D^{\mu} e(t)
$$

where, $k_{p}$ is the proportional term, $k_{i}$ is the integral time term, $k_{d}$ is the derivative time term, $\lambda$ is the non-integer order of the integral term, and $\mu$ is the non-integer order of the derivative term. Assuming $e(t)=\tilde{q}$, the new control law for the linearized system (4) with the FOPID controller (10) is:

$a=\ddot{q}_{d}-k_{p} \tilde{q}-k_{i} \frac{d^{-\lambda}}{d t} \tilde{q}-k_{d} \frac{d^{\mu}}{d t} \tilde{q}$

Replacing (11) in (4):

$$
\tilde{q}+k_{p} \tilde{q}+k_{i} \frac{d^{-\lambda}}{d t} \tilde{q}+k_{d} \frac{d^{\mu}}{d t} \tilde{\dot{q}}=0
$$

Passing (12) to the Laplace domain, the desired characteristic polynomial is obtained:

$s^{2}+k_{p}+\frac{k_{i}}{s^{\lambda}}+k_{d} s^{\mu}=0$

From (13), the equation contains the FractionalOrder terms $(\lambda, \mu)$ that rise to five the degree of freedom of the characteristic polynomial of the system. Therefore, a solution based in optimization algorithms is proposed to find the FOPID controller terms. This methodology employs the non-linear conditions (14)-(18) that describes the desired behavior of the system in the frequency domain to reach a robust performance and stability.

Phase margin $(p)$ :

$\arctan \left(C\left(j w_{c}\right) P\left(j w_{c}\right)\right)=-180+p m$

Gain crossover frequency $\left(w_{c}\right)$ :

$$
\left|C\left(j w_{c}\right) P\left(j w_{c}\right)\right|=0 d B
$$

Robustness against plant gain variations:

$$
\frac{d}{d w}\left(\arctan \left(C\left(j w_{c}\right) P\left(j w_{c}\right)\right)\right)=0
$$

High frequency noise rejection:

$$
\left|\frac{C\left(j w_{c}\right) P\left(j w_{c}\right)}{1+C\left(j w_{c}\right) P\left(j w_{c}\right)}\right|=M_{t} d B
$$

Output disturbance rejection:

$$
\left|\frac{1}{1+C\left(j w_{c}\right) P\left(j w_{c}\right)}\right|=M_{s} d B
$$

where $C\left(j w_{c}\right)$ is the FOPID controller transfer function, $P\left(j w_{c}\right)$ is the system transfer function, $p m$ is the phase margin, $w_{c}$ is the gain crossover frequency, $M_{s}$ and $M_{t}$ are the maximum values for the sensitivity and complementary sensitivity functions. MATLAB FMINCON algorithm is employed to solve the multiobjective optimization problem. the cost function for the FOPID controller tuning, is given by (14), the optimization constrains are (15)-(18), and the ISE criterion is selected as performance index for the algorithm.

\section{CASE STUDY: TWO DEGREE OF FREEDOM ROBOTIC MANIPULATOR}

As shown in Fig.1, the two degree of freedom robotic manipulator moves through the plane $x_{0}-$ $y_{0}$. As can be observed, $q_{1}$ is the movement of the first link with length $l_{1}$, measured with reference to the coordinate system $x_{0}-y_{0}$. On the other hand, 
$q_{2}$ is the movement of the second link with length $l_{2}$ measured with reference to the coordinate system $x_{1}-y_{1}$. Besides, the fixed reference system $\sum_{0}\left(x_{0}, y_{0}\right)$ is in the joint of the first link, making $z_{0}$ the rotation axis of the manipulator. As well, the reference system $\sum_{1}\left(x_{1}, y_{1}\right)$ is in the joint of the second link, and the reference system $\sum_{2}\left(x_{2}, y_{2}\right)$ is in the final effector of the manipulator. The gravity vector $g$ is in parallel to the axis $y_{0}$ of the reference system $\sum_{0}\left(x_{0}, y_{0}\right)$.

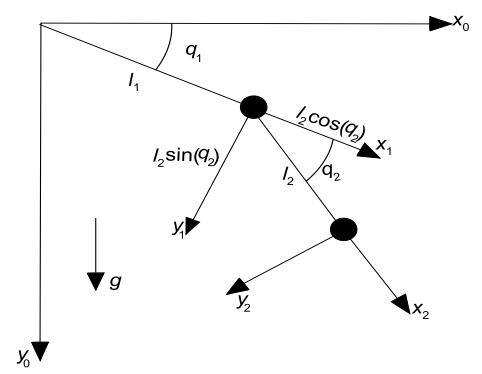

Figure 1. Two degree of freedom robotic manipulator

Applying the homogeneous transformation matrices to the robotic system links, the direct kinematic model of the system is given by (19). The inverse kinematics of the robotic manipulator is presented in (20), which is obtained from the algebraic analysis of (19).

$$
\left[\begin{array}{l}
x_{0} \\
y_{0}
\end{array}\right]=\left[\begin{array}{c}
l_{1} \sin \left(q_{1}\right)+l_{2} \sin \left(q_{1}+q_{2}\right) \\
-l_{1} \cos \left(q_{1}\right)+l_{2} \cos \left(q_{1}+q_{2}\right)
\end{array}\right]
$$

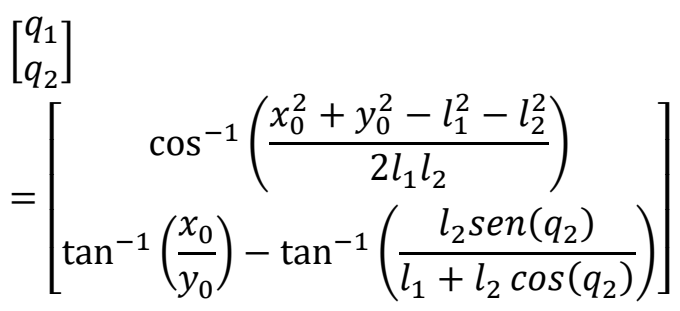

From [17], the Dynamic model of any robotic system is given by (1), where this model can be obtained by the Euler-Lagrange method, the virtual work principle, and the Newton-Euler recursive method. Applying the Euler-Lagrange method, the dynamic model of the two degree of freedom robotic manipulator is given by (21), where $\tau_{1}$ and $\tau_{2}$ are the applied torque to the joints $q_{1}$ and $q_{2}, m_{1}$ and $m_{2}$ are the links mass, $l_{c 11}$ and $l_{c 12}$ indicate the position of the gravity center of each link, $g$ is the gravity vector, $I_{1}$ and $I_{2}$ are the links inertia, $q_{1}, q_{2}, \dot{q_{1}}, \dot{q_{2}}, \ddot{q}_{1}$ and $\ddot{q}_{2}$ are the position, velocity and acceleration of each link respectively. Finally, $b_{1}, b_{2}$ and $f_{c 1}, f_{c 2}$ are the viscous and Coulomb frictions of each link

$$
\begin{aligned}
\tau_{1}=\left[m_{1} l_{c 1}^{2}+m_{2}\right. & l_{1}^{2}+m_{2} l_{c 2}^{2} \\
& +2 m_{2} l_{1} l_{c 2} \cos \left(q_{2}\right)+I_{1} \\
& \left.+I_{2}\right] \ddot{q}_{1}+\left[m_{2} l_{c 2}^{2}\right. \\
& \left.+2 m_{2} l_{1} l_{c 2} \cos \left(q_{2}\right)+I_{2}\right] \ddot{q}_{2} \\
& -2 l_{1} m_{2} l_{c 2}^{2} \sin \left(q_{2}\right) \dot{q}_{1} \\
& -l_{1} m_{2} l_{c 2}^{2} \sin \left(q_{2}\right) \dot{q}_{2} \\
& +g\left(l_{c 1} m_{1}+m_{2} l_{1}\right) \sin \left(q_{1}\right) \\
& +g m_{2} l_{c 2} \sin \left(q_{1}+q_{2}\right) \\
& +b_{1} \dot{q}_{1}+f_{c 1} \operatorname{sign}\left(\dot{q}_{1}\right) \\
& +f_{e 1}\left[1-\left|\operatorname{sign}\left(\dot{q}_{1}\right)\right|\right]
\end{aligned}
$$

$$
\begin{aligned}
\tau_{2}=\left[m_{2} l_{c 2}^{2}+I_{2}\right. & \left.+l_{1} m_{2} l_{c 2}^{2} \cos \left(q_{2}\right)\right] \ddot{q}_{1} \\
& +\left[m_{2} l_{c 2}^{2}+I_{2}\right] \ddot{q}_{2} \\
& -2 l_{1} m_{2} l_{c 2}^{2} \sin \left(q_{2}\right) \dot{q}_{1} \\
& +g m_{2} l_{c 2} \sin \left(q_{1}+q_{2}\right) \\
& +b_{2} \dot{q}_{2}+f_{c 2} \operatorname{sign}\left(\dot{q}_{2}\right) \\
& +f_{e 2}\left[1-\left|\operatorname{sign}\left(\dot{q}_{2}\right)\right|\right]
\end{aligned}
$$

. For the identification process, the dynamic model of the robotic manipulator (21) is parametrized in the matrix form (1) as shown in (22).

$$
\begin{gathered}
M(q)=\left[\begin{array}{cc}
\theta_{1}+2 \theta_{2} \cos \left(q_{2}\right) & \theta_{3}+\theta_{2} \cos \left(q_{2}\right) \\
\theta_{3}+\theta_{2} \cos \left(q_{2}\right) & \theta_{3}
\end{array}\right] \\
C(q, \dot{q})=\left[\begin{array}{cc}
-2 \theta_{2} \sin \left(q_{2}\right) \dot{q}_{1} & -\theta_{2} \sin \left(q_{2}\right) \dot{q}_{2} \\
\theta_{2} \sin \left(q_{2}\right) \dot{q}_{1} & 0
\end{array}\right] \\
g(q)=\left[\begin{array}{c}
\theta_{4} \sin \left(q_{1}\right)+\theta_{5} \sin \left(q_{1}+q_{2}\right) \\
\theta_{5} \sin \left(q_{1}+q_{2}\right)
\end{array}\right]
\end{gathered}
$$




$$
\begin{aligned}
& f\left(\dot{q}, f_{c}\right) \\
& =\left[\begin{array}{l}
\theta_{6} \dot{q}_{1}+\theta_{8} \operatorname{sign}\left(\dot{q}_{1}\right)+\theta_{10}\left[1-\left|\operatorname{sign}\left(\dot{q}_{1}\right)\right|\right] \\
\theta_{7} \dot{q}_{2}+\theta_{9} \operatorname{sign}\left(\dot{q}_{2}\right)+\theta_{11}\left[1-\left|\operatorname{sign}\left(\dot{q}_{2}\right)\right|\right]
\end{array}\right]
\end{aligned}
$$

where the $\theta$ parameters are:

$$
\begin{aligned}
& \theta_{1}=m_{1} l_{c 1}^{2}+m_{2} l_{1}^{2}+m_{2} l_{c 2}^{2}+I_{1}+I_{2} \\
& \theta_{2}=l_{1} m_{2} l_{c 2}^{2} ; \theta_{3}=m_{2} l_{c 2}^{2}+I_{2} ; \\
& \theta_{4}=g\left(l_{c 1} m_{1}+m_{2} l_{1}\right) ; \theta_{5}=g m_{2} l_{c 2} \\
& \theta_{6}=b_{1} ; \theta_{7}=b_{2} ; \theta_{8}=f_{c 1} ; \theta_{9}=f_{c 2} ; \\
& \theta_{10}=f_{e 1} ; \theta_{11}=f_{e 2}
\end{aligned}
$$

Notice $\theta_{i}$ parameters will be obtained employing the identification algorithm. Notice that $\theta_{i}$ parameters (for $i=1-5$ ) are in function of the physical dimensions of the manipulator. However, the remaining parameters depends of the viscous and Coulomb frictions of each joint, and changes according to the operating condition of the robotic system. A dynamic cosimulation model of the robotic manipulator is built to analyze its dynamical behavior, which incorporates the physical effects acting on the robotic manipulator as the Viscous and Coulomb frictions and body inertias. The cosimulation model is shown in Fig.2. As can be observed, the manipulator is parallel to the gravity vector, which should be compensated by the controller. The robotic system turns around the axis, and the applied torque is perpendicular to the rotation axis. This MSC-ADAMS model is exported to MATLAB using the ADAMS-controls toolbox to perform the identification and control stages.

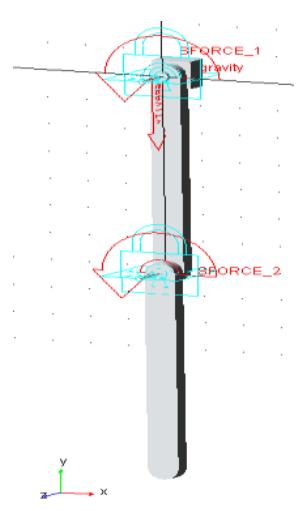

Figure 2. Two degree of freedom robotic manipulator built in MSC-ADAMS

\section{DYNAMIC MODEL PARAMETRIC IDENTIFICATION}

The recursive least squares algorithm is employed for the parametric identification of the robotic manipulator shown in Fig.2. Notice that this step is required because although the physical dimensions of the robot are well known, the Viscous and Coulomb frictions of each joint are unknown. The parametrized model of the robotic manipulator given by (22) shows the $\theta_{i}$ parameters, which are associated to non-linear functions dependent of the joint position, velocity, and acceleration of the system. However, this $\theta_{i}$ parameters can be calculated using linear regression matrices. For the multivariable case, the recursive least squares algorithm employs the linear regressor (23) described by [21] where $y_{n}(k)$ is the measured output vector, $\psi(k)$ is the regression matrix, and $\hat{\theta}$ is the vector of estimated parameters.

$\left[\begin{array}{c}y_{1}(k) \\ y_{2}(k) \\ \vdots \\ y_{n}(k)\end{array}\right]=\psi(k)^{T} \hat{\theta}(k-1)$

The recursive least squares descripted in (24) employs samples from joint position, velocity, and acceleration as the applied joint torques to the robotic system, which are taken from the MSCADAMS/MATLAB cosimulation model, where $\hat{\theta}(k)$ is the vector of estimated parameters, $\psi(k)$ is the regression matrix, $e(k)$ is the error for each iteration, and $P(k)$ is the covariance matrix.

$$
\begin{aligned}
& \hat{\theta}(k)=\hat{\theta}(k-1)+P(k-1) \Psi(\mathrm{k}) \\
& {\left[\mathrm{I}+\Psi(\mathrm{k}-1)^{\mathrm{T}} \mathrm{P}(\mathrm{k}-1) \Psi(\mathrm{k})\right]^{-1} e(k)} \\
& P(k)=P(k-1)-P(k-1) \Psi(k) \\
& {\left[I+\Psi(k)^{T} P(k-1) \Psi(k)\right]^{-1} \Psi(k)^{T} P(k-1)} \\
& e(k)=y(k)-\psi(k)^{T} \hat{\theta}(k-1)
\end{aligned}
$$

The identification trajectory selection is an important task for the parametric identification of the dynamic model of a robotic manipulator since the quality of the estimation depends of the regressor persistent excitation [22]. The selected 
trajectories (25) and (26) are shown in Fig.3 for the joints $q_{1}, q_{2}$ respectively, composed by the superposition of sinusoidal functions that ensure the regressor persistent excitation.

$$
\begin{gathered}
P_{1}(t)=120\left(1-e^{-0.8 t}\right) \\
+35(\sin (20 t+0.15) \\
+\sin (16 t+0.5)) \\
\\
\\
P_{2}(t)=1.2\left(1-e^{-1.8 t}\right)+\sin (36 t+0.3) \\
+0.25 \sin (12 t+0.34)
\end{gathered}
$$

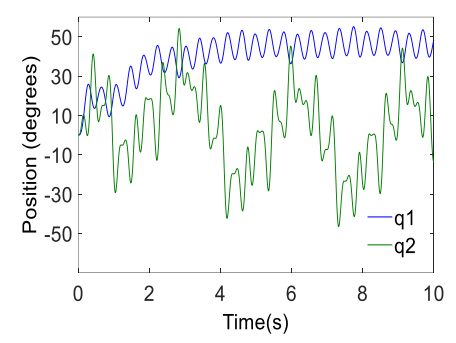

Figure 3. Proposed identification trajectories for $q_{1}$ and $q_{2}$

Applying the trajectories (25) and (26) to the MSC-ADAMS / MATLAB co-simulation model of the manipulator, the joint position, velocity, acceleration, and joint applied torque data are obtained. These data are entered in the recursive least squares algorithm (24), obtaining the parameters of the dynamic model (22), which are $\theta_{1}=1.79, \quad \theta_{2}=0.29, \quad \theta_{3}=0.18, \quad \theta_{4}=61.80$, $\theta_{5}=6.69, \quad \theta_{6}=-3.28, \quad \theta_{7}=0.040, \quad \theta_{8}=$ $-0.3114, \theta_{9}=0.0021, \theta_{10}=\theta_{11}=0$.

To validate the identified dynamic model of the robotic manipulator, the applied joint torque from the MSC-ADAMS/MATLAB cosimulation model is compared with the applied torque from the dynamic model (22) built in SIMULINK, which employs the estimated $\theta_{i}$ parameters presented in Table I. Figure 4 Shows the applied joint torque of the cosimulation model versus the applied joint torque of the identified dynamic model. As can be observed, the applied torque from the identified dynamic model is like the MSC-ADAMS/MATLAB cosimulation model. The RMSE value given by (27) is employed as fit measure between the MSC-
ADAMS/MATLAB cosimulation model and the identified dynamic model, where $T$ is the total amount of samples, and $e(t)$ is the error between the applied torques. The RMSE values for $q_{1}, q_{2}$ are 0.0182 and 0.0018 . So that, it can be said that the identified model approaches the dynamic model of the robotic manipulator.

$$
R M S E=\sqrt{\frac{1}{T} \int_{0}^{T} e(t)^{2} d t}
$$

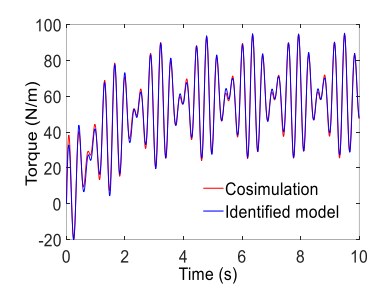

(a)

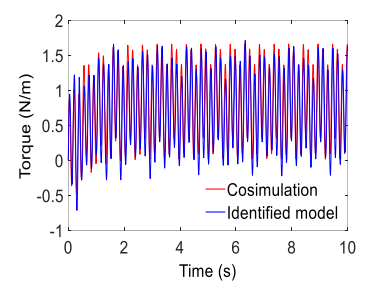

(b)
Figure 4. Applied torque validation from (a) MSCADAMS/MATLAB cosimulation model Identified dynamic model

\section{FOPID AND IOPID CONTROLLER DESIGN}

The IOPID and FOPID controllers with the computed torque control are tuned to reach a robust performance and stability. Hence, the desired operating conditions consider a time response without overshoot $(\zeta=1)$ or a $p m=100^{\circ}$, and a settling time of the system of $t_{s}=0.2 \mathrm{~s}$, which requires a $w_{n}=20 \mathrm{rad} / \mathrm{s}$. Applying the tuning methodologies presented in Section II and Section III, the obtained terms for the IOPID and FOPID controllers are presented in Table I.

TABLE I

\section{IOPID AND FOPID CONTROLLER CONSTANTS}

\begin{tabular}{cccccc} 
Controller & $\boldsymbol{k}_{\boldsymbol{p}}$ & $\boldsymbol{k}_{\boldsymbol{i}}$ & $\boldsymbol{k}_{\boldsymbol{d}}$ & $\boldsymbol{\lambda}$ & $\boldsymbol{\mu}$ \\
\hline IOPID & 440 & 400 & 40 & 1 & 1 \\
\hline FOPID & 729 & 0.2 & 100 & 0.85 & 0.84 \\
\hline
\end{tabular}

The two degrees of freedom robotic manipulator is tested for tracking tasks in order to evaluate the IOPID and FOPID controllers performance. Fig.5 shows the proposed desired trajectory for a specific task, which employs a cartesian trajectory planner to 
set the trajectory for each joint of the robot. Thus, the final effector of the robot can be carried from an initial point to a final point through a straight line under certain velocity, acceleration, and Jerk conditions, considering the physical actuators restrictions.

The trajectory planner is formed by three segments descripted by a 6-1-6 polynomial [23]. The first segment is the acceleration section and is represented for a sixth order polynomial. The second segment is the constant velocity section and is represented with a first order polynomial. The third segment belongs to the deceleration segment and is represented by a sixth order polynomial. Notice the first and third segments employs a high order polynomial to reach a smooth jerk variation.

The spatial trajectory produced by the final effector employing the IOPID and FOPID controllers with the computed torque control strategy is presented in Fig.5. As can be observed, the trajectory that corresponds to a triangular shape, shows that the robotic manipulator has a good performance for tracking tasks using the IOPID and FOPID controllers.

The position error for each joint employing the IOPID and FOPID controllers is presented in Fig.6, which shows that the FOPID controller with computed torque control has a less joint error than the IOPID controller with the computed torque control. The control action is presented in Fig.7, where the magnitude of the control action is similar for the IOPID and FOPID controllers, indicating that both the FOPID controller and the IOPID controller requires the same energy to reach the desired trajectory.

\section{CONTROL PERFORMANCE ASSESSMENT}

Three tests are performed to evaluate the IOPID and FOPID controllers robustness. On the first test, a step-type external disturbance of $0.1 s$ is added to each joint torque while the robotic manipulator performs the desired trajectory presented in Fig.6. For the joint $q_{1}$ the disturbance magnitude is $150 \mathrm{~N} / \mathrm{mm}$ and $25 \mathrm{~N} / \mathrm{mm}$ for the joint $q_{2}$. Figures 9 to 10 present the spatial performance and the joint error of the robotic system. As can be observed, the external disturbances affect the desired trajectory; however, the IOPID and FOPID controllers return the robotic system to the desired path.

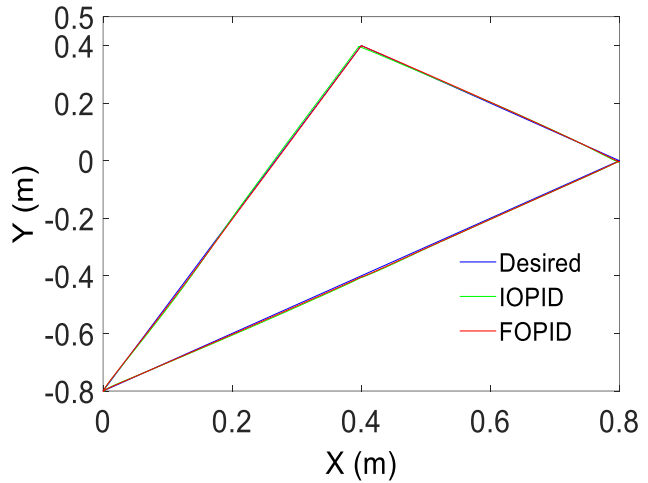

Figure 6. Tracking performance of the IOPID and FOPID

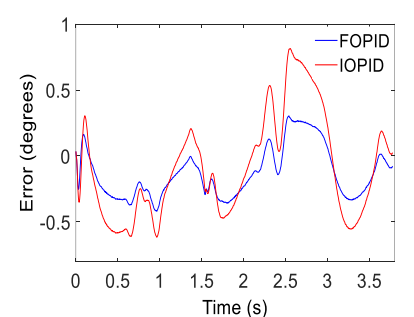

(a)

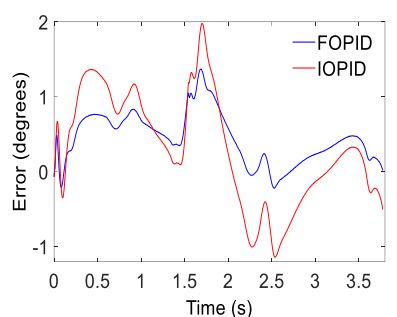

(b)
Figure 6. Joint error using the IOPID and FOPID controllers for (a) $q_{1}$ and (b) $q_{2}$.

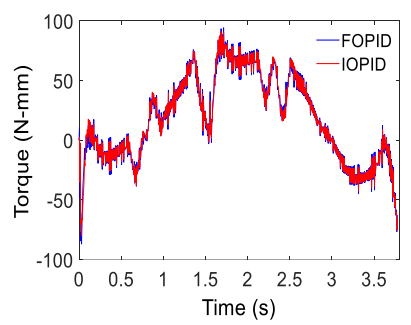

(a)

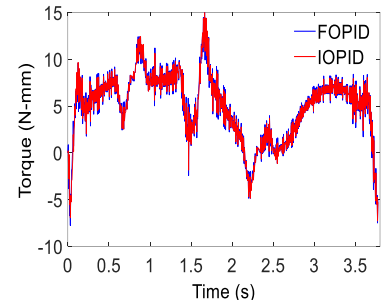

(b)
Figure 7. Joint applied torque using the IOPID and FOPID controllers for (a) $q_{1}$ and (b) $q_{2}$.

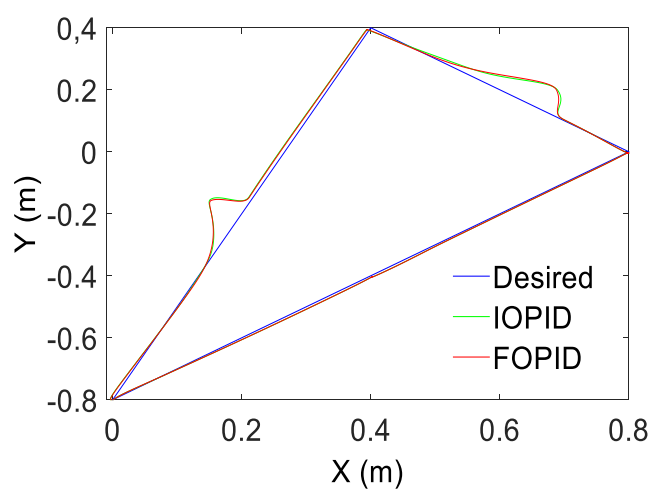

Figure 8 . Tracking performance of the IOPID and FOPID controllers in presence of external disturbance. 


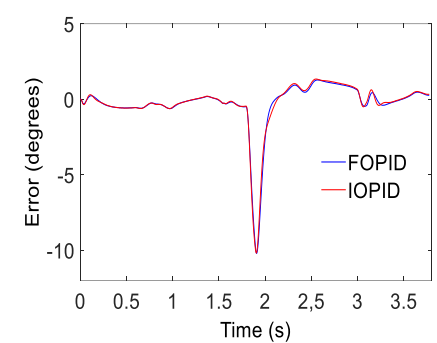

(a)

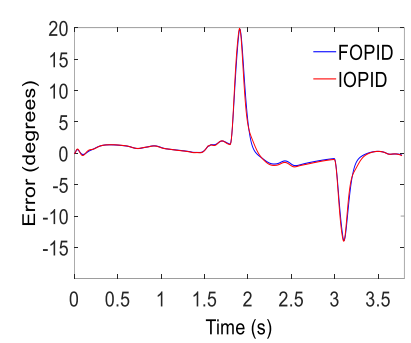

(b)
Figure 9. Joint error in presence of external disturbances using the IOPID and FOPID controllers for (a) $q_{1}$ and (b) $q_{2}$.

For the second test, the robotic system payload is varied to evaluate the performance of the IOPID and FOPID controllers. Hence, an $2 \mathrm{~kg}$ payload is placed in the final effector of the robotic manipulator while it performs the desired trajectory, which response is presented in Fig.10. As can be observed, despite the payload variation, the FOPID controller has a better tracking response than the IOPID controller. Likewise, Fig.11 presents the joint error in $q_{1}$ and $q_{2}$ for the IOPID and the FOPID controllers. As can be observed, the highest joint error in $q_{1}$ and $q_{2}$ coincide with the trajectory point $(0.8,0)$ where $q_{1}$ and $q_{2}$ are perpendicular to the gravity vector. However, the joint error is less when the FOPID controller is employed, indicating a better performance for payload variations. The third test introduces a random noise with \pm 5 degrees amplitude in the feedback loop of each joint of the robotic system. Fig.13 shows the effect of the random noise while the robotic manipulator performs the desired trajectory employing the IOPID and FOPID controllers. As can be observed, both the IOPID and the FOPID controllers are affected by the presence random noise in the feedback loop. Fig.14 presents the joint error in $q_{1}$ and $q_{2}$ when the IOPID and FOPID controllers are employed in presence of random noise in the feedback loop. As can be appreciated, the joint error rises significantly for $q_{1}$ and $q_{2}$, especially when the IOPID controller is employed.

The tracking norm $N$ (28) proposed by [24] is employed to stablish a quantitative comparison between the IOPID and the FOPID controllers performance for each joint of the robotic system

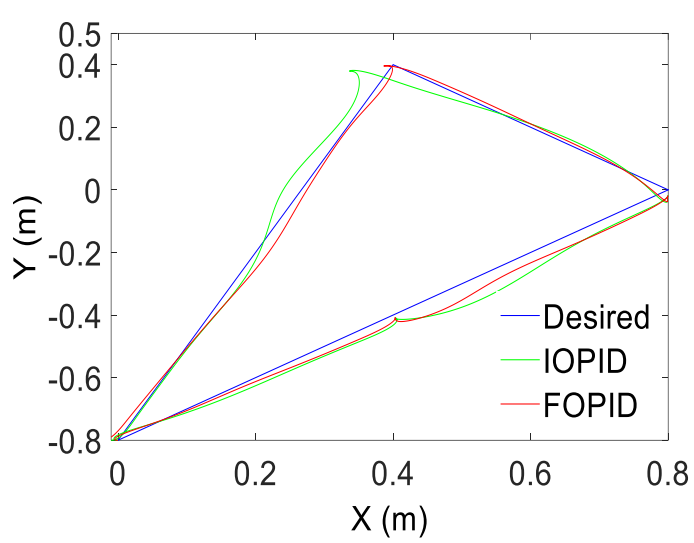

Figure 10. Tracking performance of the IOPID and FOPID controllers with payload variations

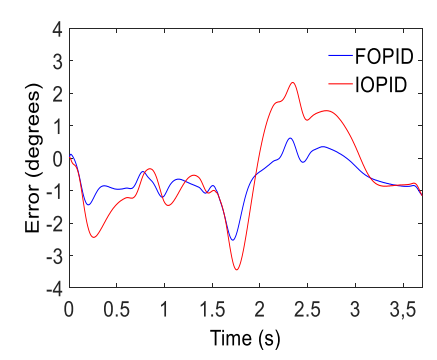

(a)

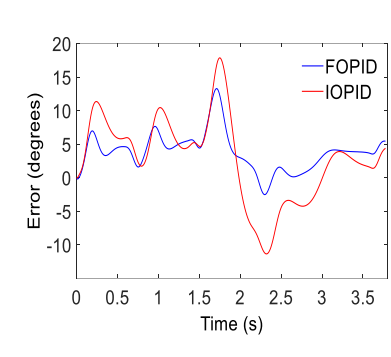

(b)
Figure 11. Joint error in presence of payload variations employing the IOPID and FOPID controllers for (a) q1 and (b) q2

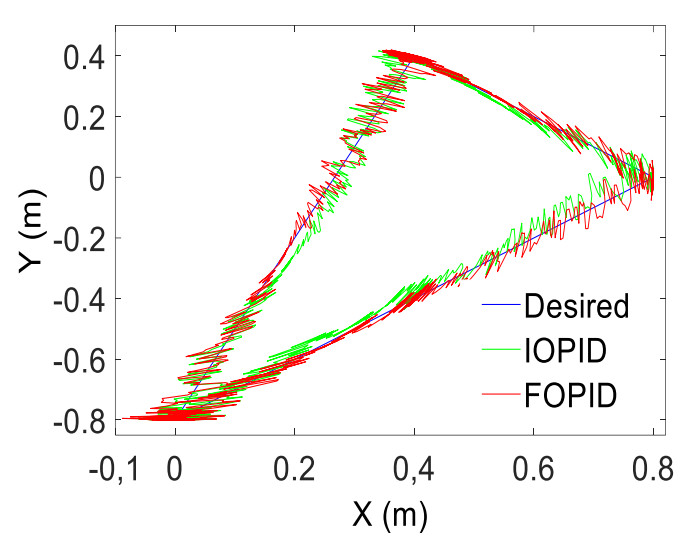

Figure 13. Tracking performance of the IOPID and FOPID controllers in presence of random noise in the feedback loop

$$
N=\sqrt{\frac{1}{T} \int_{0}^{T} \tilde{q}(t)^{2}+\dot{\tilde{q}}(t)^{2} d t}
$$




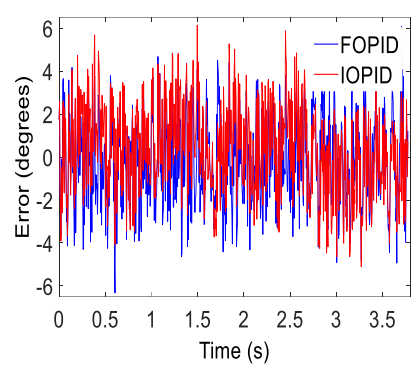

(a)

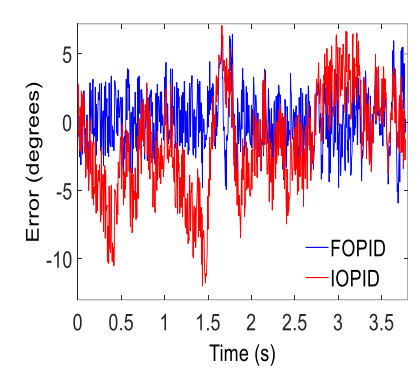

(b)
Figure 14. Joint error in presence of random noise in the feedback loop using the IOPID and FOPID controllers for (a) $q_{1}$ and (b) $q_{2}$.

where $T$ is the total time of the trajectory, $\tilde{q}(t)$ is the joint position error and $\dot{\tilde{q}}(t)$ is the joint velocity error. In addition, to obtain a general index of the tracking tasks performances, the $N_{\text {total }}$ tracking norm (29) is proposed, which is based in (28) and consider the position and velocity errors of the $n$ joints of the robotic system.

$N_{\text {total }}=\sqrt{\frac{1}{T} \int_{0}^{T} \sum_{i=1}^{n}\left(\widetilde{q}_{l}(t)^{2}+\dot{\widetilde{q}}_{l}(t)^{2}\right) d t}$

Table III summarizes the performance norms (28) and (29) for the IOPID and FOPID controllers on nominal operation conditions, presence of external disturbances in the joint torque, payload variations, and random noise in the feedback loop. As can be observed, for the nominal operation conditions, the norm $N_{q 1}$ and $N_{q 2}$ is less for the FOPID controller than for the IOPID controller, indicating FOPID controller has a better performance for tracking tasks with an improvement of $34.7 \%$.

In presence of external disturbances, Table IV shows that for the FOPID controller, the norm $N_{q 1}$ and $N_{q 2}$ is less regarding to the IOPID controller with an $46.12 \%$ improve. Therefore, it can be said that the FOPID Controller is more robust than the IOPID controller in presence of external disturbances, which proves that the FOPID controller makes a better trajectory correction when a disturbance appears.

Analyzing the robotic system performance in presence of random noise in the feedback loop, the $N_{q_{1}}$ norm is similar for the IOPID and FOPID controllers. However, for the $N_{q_{2}}$ norm a significantly improvement of $20.19 \%$ is reached. Besides, the $N_{\text {total }}$ norm indicates that the FOPID controller is $11.32 \%$ better than the IOPID controller. For this reason, although the presence of random noise in the feedback loop has a great effect over the tracking tasks, the FOPID controller has a less position and velocity errors, so employing the FOPID controller makes more robust the robotic system against the presence of random noise in the feedback loop.

Also, Table IV shows the RMS value of the applied torque of each joint of the robotic system when the IOPID and FOPID controllers with the computed torque control strategy are employed. As can be observed, the RMS value on $q_{1}$ and $q_{2}$ has similar values for the nominal operation, presence external disturbance, and payload variation tests. In contrast, for the random noise in the feedback loop test, the magnitude of the applied torque is bigger than in the previous tests because the IOPID and FOPID controllers must make a greater trajectory correction rising the applied torque in $q_{1}$ and $q_{2}$.

So that, although the applied torque required by the IOPID and FOPID controllers is similar, the FOPID controller has the better results for tracking tasks with a less position and velocity errors in presence of the analyzed disturbances.

\section{CONCLUSIONS}

This paper presented the design of a FOPID controller with the computed torque control strategy for the tracking control of a two degrees of freedom robotic manipulator. The kinematic and dynamic model of the robotic system were obtained, and the dynamic model was identified employing the recursive least squares algorithm. The proposed control strategy was contrasted with a IOPID controller with the computed torque control strategy in presence of external disturbances, payload variations and random noise in the feedback loop.

Obtained results from the robustness analysis shows that the FOPID controller has a better performance for tracking tasks since FOPID controller has less position error and velocity error than the IOPID controller. In addition, the FOPID controller has a better external disturbance rejection with a $46.12 \%$ improvement, a better performance for payload variations with a $31.59 \%$ improvement, and a better response to the presence of random noise in the feedback loop with $11.32 \%$ improvement. Also, there is not significantly differences for the proposed tests regarding to the applied joint torque. For these reasons, it can be said that the FOPID controller is more robust than the IOPID controller to perform tracking tasks. 
TABLE II TRACKING NORMS N OF THE ROBOTIC SYSTEM JOINTS IN TRACKING TASKS FOR NOMINAL OPERATION CONDITIONS AND THE PRESENCE OF EXTERNAL DISTURBANCES

\begin{tabular}{|c|c|c|c|c|c|c|c|c|c|c|c|c|}
\hline \multirow{2}{*}{$\begin{array}{c}\text { Tracking } \\
\text { norm }\end{array}$} & \multicolumn{3}{|c|}{ Nominal operation } & \multicolumn{3}{|c|}{ External disturbance } & \multicolumn{3}{|c|}{ Payload variation } & \multicolumn{3}{|c|}{ Random noise } \\
\hline & FOPID & IOPID & $\begin{array}{c}\text { Improve } \\
(\%)\end{array}$ & FOPID & IOPID & $\begin{array}{c}\text { Improve } \\
(\%)\end{array}$ & FOPID & IOPID & $\begin{array}{c}\text { Improve } \\
(\%)\end{array}$ & FOPID & IOPID & $\begin{array}{c}\text { Improve } \\
(\%)\end{array}$ \\
\hline$N_{q_{1}}$ & 0.23 & 0.41 & 43.82 & 0.98 & 1.76 & 44.32 & 0.90 & 1.41 & 36.50 & 14.90 & 14.83 & -0.47 \\
\hline$N_{q_{2}}$ & 0.57 & 0.84 & 32.12 & 2.22 & 4.15 & 46.51 & 4.74 & 6.92 & 31.50 & 14.47 & 18.13 & 20.19 \\
\hline$N_{\text {total }}$ & 0.61 & 0.93 & 34.70 & 2.43 & 4.51 & 46.12 & 4.83 & 7.06 & 31.59 & 20.77 & 23.42 & 11.32 \\
\hline
\end{tabular}

TABLE III RMS VALUE OF THE ROBOTIC SYSTEM JOINTS IN TRACKING TASKS FOR NOMINAL OPERATION CONDITIONS AND THE PRESENCE OF EXTERNAL DISTURBANCES

\begin{tabular}{|c|c|c|c|c|c|c|c|c|}
\hline \multirow{2}{*}{ Joint } & \multicolumn{2}{|c|}{ Nominal operation } & \multicolumn{2}{|c|}{ External disturbance } & \multicolumn{2}{|c|}{ Payload variation } & \multicolumn{2}{|c|}{ Random noise } \\
\hline & FOPID & IOPID & FOPID & IOPID & FOPID & IOPID & FOPID & IOPID \\
\hline$q_{1}$ & 40.05 & 39.92 & 43.3 & 43.6 & 44.52 & 44.49 & 659 & 623 \\
\hline$q_{2}$ & 6.12 & 6.11 & 6.73 & 6.8 & 13.14 & 12.98 & 132 & 124 \\
\hline
\end{tabular}

\section{CONCLUSIONS}

This paper presented the design of a FOPID controller with the computed torque control strategy for the tracking control of a two degrees of freedom robotic manipulator. The kinematic and dynamic model of the robotic system were obtained, and the dynamic model was identified employing the recursive least squares algorithm. The proposed control strategy was contrasted with a IOPID controller with the computed torque control strategy in presence of external disturbances, payload variations and random noise in the feedback loop.

Obtained results from the robustness analysis shows that the FOPID controller has a better performance for tracking tasks since FOPID controller has less position error and velocity error than the IOPID controller. In addition, the FOPID controller has a better external disturbance rejection with a $46.12 \%$ improvement, a better performance for payload variations with a $31.59 \%$ improvement, and a better response to the presence of random noise in the feedback loop with $11.32 \%$ improvement. Also, there is not significantly differences for the proposed tests regarding to the applied joint torque. For these reasons, it can be said that the FOPID controller is more robust than the IOPID controller to perform tracking tasks.

\section{References:}

[1] J. Yoo, I. W. Park, V. To, J. Q. H. Lum, and T. Smith, "Avionics and perching systems of free-flying robots for the International Space Station," in Systems Engineering (ISSE), 2015 IEEE International Symposium on, 2015, pp. 198-201.

[2] R. M. Muller, "Assembly and servicing of a large telescope at the International Space Station," in Aerospace Conference Proceedings, 2002. IEEE, 2002, vol. 7, pp. 7-3611-7-3619 vol.7.

[3] M. A. Diftler et al., "Robonaut 2 \#x2014; Initial activities on-board the ISS," in Aerospace Conference, 2012 IEEE, 2012, pp. 1-12.

[4] P. Fairley, "Robot miners of the briny deep," 
IEEE Spectr., vol. 53, no. 1, pp. 44-47, 2016.

[5] R. Saltaren, R. Aracil, C. Alvarez, E. Yime, and J. M. Sabater, "Field and service applications - Exploring deep sea by teleoperated robot - An Underwater Parallel Robot with High Navigation Capabilities," IEEE Robot. Autom. Mag., vol. 14, no. 3, pp. 65-75, Sep. 2007.

[6] S. Karande, P. S. V Nataraj, P. S. Gandhi, and M. M. Deshpande, "Control of parallel flexible five bar manipulator using QFT," in Industrial Technology, 2009. ICIT 2009. IEEE International Conference on, 2009, pp. $1-6$.

[7] R. Comasolivas, J. Quevedo, T. Escobet, A. Escobet, and J. Romera, "Low level control of an omnidirectional mobile robot," in Control and Automation (MED), 2015 23th Mediterranean Conference on, 2015, pp. 1160-1166.

[8] G. Rigatos and P. Siano, "An H-infinity feedback control approach to autonomous robot navigation," in Industrial Electronics Society, IECON 2014 - 40th Annual Conference of the IEEE, 2014, pp. 26892694.

[9] Y. Miyasato, "Adaptive H-infinity; control of nonholonomic mobile robot based on inverse optimality," in American Control Conference, 2008, 2008, pp. 3524-3529.

[10] A. Tayebi, S. Abdul, M. B. Zaremba, and Y. Ye, "Robust Iterative Learning Control Design: Application to a Robot Manipulator," IEEE/ASME Trans. Mechatronics, vol. 13, no. 5, pp. 608-613, Oct. 2008.

[11] N. Pons, G. Bourdon, and S. Delaplace, "Contribution of fuzzy and feedback control to a mobile robot adaptative navigation," in Control Applications, 1994., Proceedings of the Third IEEE Conference on, 1994, pp.
65-69 vol.1.

[12] R. Lin and T. Namerikawa, "Robust control of master-slave robot system considering environmental uncertainties," in Advanced Intelligent Mechatronics. Proceedings, 2005 IEEE/ASME International Conference on, 2005, pp. 1299-1304.

[13] J. Viola and L. Angel, "Identification, control and robustness analysis of a robotic system using fractional control," Lat. Am. Trans. IEEE (Revista IEEE Am. Lat., vol. 13, no. 5, pp. 1294-1302, May 2015.

[14] F. Jiahe and L. Rui, "Fractional PID and backstepping control for a small quadrotor helicopter," in Control Conference (CCC), 2015 34th Chinese, 2015, pp. 5701-5706.

[15] Q. Xu, J. Huang, and L. Zhou, "ANNinversion based fractional-order sliding control for the industrial robot," in Control Conference (CCC), 2015 34th Chinese, 2015, pp. 4501-4505.

[16] Y. Zennir, A. Makhbouche, L. Bouras, and R. Bendib, "Fractional order multi-controller design to control a manipulator robot wrist," in 2015 4th International Conference on Electrical Engineering (ICEE), 2015, pp. 16.

[17] R. Kelly, V. S. Davila, and J. A. L. Perez, Control of Robot Manipulators in Joint Space. Springer London, 2006.

[18] I. Podlubny, Fractional Differential Equations: An Introduction to Fractional Derivatives, Fractional Differential Equations, to Methods of Their Solution and Some of Their Applications. Academic Press, 1999.

[19] K. B. Oldham and J. Spanier, The fractional calculus: theory and applications of differentiation and integration to arbitrary order. New York: Academic Press, 1974. 
[20] C. A. Monje, Y. Q. Chen, B. M. Vinagre, D. Xue, and V. Feliu-Batlle, Fractional-order Systems and Controls: Fundamentals and Applications. Springer London, 2010.

[21] S. P. Lloyd, "Least Squares Quantization in PCM," IEEE Trans. Inf. Theory, vol. 28, no. 2, pp. 129-137, 1982.

[22] F. R. Cortés, Matlab aplicado a robótica y mecatrónica, 1st ed. Alfaomega grupo editor, 2012.

[23] L. Angel, J. Bermudez, and O. Munoz, "Dynamic optimization and building of a parallel delta-type robot," 2013 IEEE Int. Conf. Robot. Biomimetics, ROBIO 2013, no. July, pp. 444-449, 2013.

[24] F. R. Cortés, Robotica: Control de Robots Manipuladores. Alfaomega grupo editor, 2011.

\section{Contribution of individual authors to the creation of a scientific article (ghostwriting policy)}

Jairo Viola and Luis Angel performed the controller design, robotic system modelling, simulation and performance assessment, as well as the writing for this paper.

\section{Creative Commons Attribution \\ License 4.0 (Attribution 4.0 \\ International, CC BY 4.0)}

This article is published under the terms of the Creative Commons Attribution License 4.0

https://creativecommons.org/licenses/by/4.0/deed.en US 